\title{
The effect of spiritual education on anxiety, depression and glycemic control in type 2 diabetic patients: a randomized controlled study
}

\author{
SHAHPAR BAGHERI ${ }^{1, A, E, F}$, MINA MOSALLANEZHAD², B, c, F, \\ ORCID ID: 0000-0003-4100-9901 \\ FATEME BARZGAR ${ }^{2, \mathrm{~B}, \mathrm{c}, \mathrm{F}}, \mathrm{AZADEH} \mathrm{AMIRI}^{3, \mathrm{~A}, \mathrm{c}-\mathrm{F}}$ \\ ORCID ID: 0000-0003-3501-8078
}

${ }^{1}$ Community Based Psychiatric Care Research Center, Department of Nursing, School of Nursing and Midwifery, Shiraz University of Medical Sciences, Shiraz, Iran

${ }^{2}$ Student Research Committee, Department of Surgical Technologists, School of Nursing and Midwifery Shiraz University of Medical Science, Shiraz, Iran

${ }^{3}$ Student Research Committee, School of Nursing and Midwifery, Shiraz University of Medical Sciences, Shiraz, Iran

A - Study Design, B - Data Collection, C - Statistical Analysis, D - Data Interpretation, E - Manuscript Preparation, F - Literature Search, G - Funds Collection

Summary Background. Common mental and psychological disorders in people with diabetes include anxiety and depression, which strongly affect the lives of diabetic patients and management of this disease.

Objectives. We aimed to evaluate whether spiritual education has any impact on depression and anxiety disorders and blood sugar control in patients with type 2 diabetes mellitus.

Material and methods. A randomized control trial was carried out on 146 patients with type 2 diabetes mellitus who referred to the diabetes outpatient services of the Imam Reza Clinic in Shiraz. Eligible patients were randomly assigned to the experimental group (73 subjects) and control group (73 subjects). The subjects in the experimental group were trained over four 45 -minute sessions for two weeks, and they practiced daily for four months. The control group received routine clinical care. Anxiety and depression were measured by validated questionnaires. The $\mathrm{HbA}_{1 c}$ index was also used to evaluate the control of blood sugar.

Results. The results of the study showed a significant reduction in the mean of overt anxiety $(p<0.001)$ and covert anxiety $(p<0.001)$ in depression $(p<0.001)$ and hemoglobin $\mathrm{HbA}_{1 \mathrm{c}}(p<0.001)$ in the experimental group during the study. There was no difference in the control group.

Conclusions. Spiritual training for patients with type 2 diabetes mellitus can lead to a reduction in anxiety and depression. Therefore, it is recommended that spiritual training should be used as an intervention to reduce anxiety and depression and control blood sugar in patients with type 2 diabetes mellitus in addition to clinical interventions.

Key words: type 2 diabetes mellitus, spirituality, spiritual therapies, anxiety, depression, blood glucose.

Bagheri S, Mosallanezhad M, Barzgar F, Amiri A. The effect of spiritual education on anxiety, depression and glycemic control in type 2 diabetic patients: a randomized controlled study. Fam Med Prim Care Rev 2021; 23(3): 284-289, doi: https://doi.org/10.5114/ fmpcr.2021.108190.

\section{Background}

Diabetes mellitus is one of the most common diseases which significantly affects a person's life due to its chronic nature [1]. This disease affects the lives of many people around the world. The prevalence of diabetes in 2017 was 451 million in the age group 18-99 years and is predicted to reach 693 million by 2045 [2]. Statistics show the high prevalence of the disease and its high growth in Iran. According to research conducted in 2011, more than four million Iranian adults had diabetes mellitus [3]. As soon as the disease is diagnosed, psychological and social pressures appear. One of the most significant psychological pressures for those with diabetes is depression and anxiety [4]. Globally, it is estimated that $5 \%$ of adults suffer from depression. This complication is significant in patients with diabetes. According to statistics, one in four patients with diabetes has depression with clinical symptoms [5]. These effects are mainly caused by decreased metabolic control, reduced quality of life, lack of adherence to proper diet, increased health care costs, re- duced drug adherence and high mortality, and these factors also exacerbate depression [6]. Conversely, treatment of depression with counseling and medication has noticeable effects on blood sugar level [5]. The results of the studies indicate an increased risk of depression and anxiety in diabetic patients [7]. Patients with anxiety disorders and depression go through more unfavorable periods of the disease and are more likely to become chronic and have reduced performance [8]. Research has shown that anxiety and depression affect the severity of type 2 diabetes mellitus through reduced quality of life and self-care [9]. The mortality of diabetics who also suffer from depression and anxiety is higher than those only dealing with diabetes [9]. According to research, spirituality intervention has a positive effect on a patient's mood and mental health [10] and it is an beneficial for patients with chronic disorders [11]. Diabetes patients define spirituality as a connection with one's inner self, existence of meaning in life and a connection with others, nature and a higher being (God) [12]. Studies have shown that people with chronic diseases such as diabetes use psychological support 
like spirituality as a way to deal with illness, create a sense of meaning and purpose in life, reduce emotions of suffering from illness and despair and improve self-care and management of disease-related problems $[13,14]$. It has also been shown that spirituality is a mechanism to combat the disease and is related to the control of a patient's blood sugar level [15]. Religion and spirituality, according to their internal teachings, play a role in improving the self-management function of diabetic patients $[12,16]$. This article is an attempt to examine and provide a solution to reduce psychological pressure on the patient.

\section{Objectives}

This study was conducted to evaluate whether spiritual education has any impact on depression and anxiety disorders and blood sugar control in patients with type 2 diabetes mellitus.

\section{Material and methods}

\section{Study design}

This study is a random control test with a control group and a pre- and post-intervention experimental group, which was performed after receiving approval from the ethics committee of the Shiraz University of Medical Sciences between January 2018 and June 2019. Written consent was obtained from the participants.

\section{Setting}

This study was conducted within the diabetes outpatient services of the Imam Reza Clinic, affiliated with the Shiraz University of Medical Sciences, in southern Iran.

\section{Participants}

The participants in this study, between the ages of 20 to 55 of both genders, were diagnosed with type 2 diabetes mellitus. According to a study of the effectiveness of mindfulness-based stress reduction on blood sugar control, stress, anxiety and depression in patients with type 2 diabetes mellitus [17], the required sample size was estimated at 122 people (61 people in each group). This number increased $20 \%$ to 146 people due to the probability of attrition (73 in each group). 146 people were randomly assigned to the experimental group (73 subjects) and control group (73 subjects). In the present study, the inclusion criteria were diagnosis of type 2 diabetes mellitus confirmed by a physician, not being treated by psychological medications, not suffering from severe mental illnesses, such as psychotic disorders, and not taking psychotropic drugs or substance abuse. The exclusion criteria were hospitalization due to complications of diabetes, absent more than twice in the training sessions, occurrence of unpredicted events and unwillingness of the volunteer to continue in the project.

\section{Measurement}

Data collection tools included Spielberger's covert and overt anxiety questionnaire (Spielberger State-Trait Inventory, STAI) and the Beck questionnaire. Blood samples were also taken to check blood sugar and $\mathrm{HbA}_{1 \mathrm{c}}$ under the supervision of a laboratory physician. Spielberger's overt and covert anxiety questionnaire, known as the STAI questionnaire, is a valid and reliable tool and has been used extensively in clinical research and activities [18]. The overt anxiety scale (a situational-y-1 form of STAI) consists of twenty items that evaluate a person's emotions at the time of response. Overt anxiety is scored via a Likert scale ranging from 20 to 80 and was classified into mild (20-31), moderate to low (32-42), moderate to high (43-53), relatively severe
(54-64), severe (65-75) and intense anxiety (76-80). The covert anxiety scale (Feature-form y-2 of STAI) includes twenty items that measure the individual's general and normal emotions. Covert anxiety is scored via a four-point Likert scale (never before, sometimes, most often, almost always). In this scale, scores 20-31 show mild anxiety, 32-42 moderate to low anxiety, 43-52 moderate to high anxiety, 53-62 relatively severe anxiety, 63-72 severe anxiety, and 73 or higher indicates very severe anxiety [19]. This questionnaire was translated in Iran by Mahram et al. in 1993, and its psychometrics have been confirmed [20]. Beck's depression questionnaire was first developed in 1961 by Beck et al. Due to the importance of this tool in the diagnosis of clinical intervention, many psychoanalytical studies have been conducted based on its psychometric characteristics. This questionnaire includes twenty-one questions on the Likert scale scored 1 to 63 . Scores 1-10 are normal, scores 11-16 are low depression, scores 17-20 require psychological counseling, scores $21-30$ relative depression, and scores more than 40 are excessive depression [21]. In Iran, the Beck's questionnaire psychometrics were evaluated by Tashakkori and Mehryar in 1994. Its reliability coefficient was 0.78. In radiographic studies in 1975, as well as with Wahabzadeh in 1973 and Chegini in 2002, the validity of Beck's questionnaire was proven [22]. During the treatment of diabetes, $\mathrm{HbA}_{1 \mathrm{c}}$ measurement is the best index of diabetes control. This hemoglobin shows the mean blood sugar levels over the last 8-12 weeks. Its measurement is checked once every three months. To determine $\mathrm{HbA}_{1 \mathrm{c}}$ levels, $5 \mathrm{ml}$ of blood was taken by trained personnel from the patients in the central laboratory while the patient was fasting. Patients with an index value of 4-6\% have good glycemic control, 6-8\% poor glycemic control, and more than $8 \%$ were considered to be uncontrolled [23].

\section{Intervention}

The intervention group underwent four 45-minute training sessions over two weeks. Three sections of the sessions were spiritual with religious instruction by clerics, in addition to receiving regular treatment and care. The themes of these teachings included self-knowledge and self-awareness and recognizing one's strengths and weaknesses, desires, fears, needs, tendencies. Avoid negative thoughts and try to think positively to achieve peace and a better and happier life. A session was also held by the researcher on the subject of expressing feelings and the training of problem solving. At the end, a booklet and educational CD with spiritual topics and communication with God were prepared for them. Daily practices were performed to correct emotions, control emotions, express emotions and teach relaxation and stress management. This training and practice lasted four months. The patients were followed weekly and did the daily exercises. The control group only received routine clinical treatment and care. The questionnaires were completed in two stages, one before the intervention and the other after the intervention, by the participants of the control and experimental groups.

\section{Statistical methods}

SPSS version 21 statistical software was used to analyze the data. In the present study, descriptive statistics were used to summarize the demographic characteristics in tables; to statistically analyze the data from the Kolmogorov-Smirnov tests (to check the normality assumption), we used the independent $t$-Test, paired sample test, variance analysis test for repeated measurements and Chi-square test at $\alpha=0.05$. To investigate the significant difference between the two experimental and control groups, we used the Chi-square test to evaluate the demographic information (age, work situation, education, history of sedative use). The income level was evaluated by Fisher's exact test, and age was evaluated by the independent $t$-Test. 


\section{Results}

In this study, 146 patients with type 2 diabetes mellitus were examined. Table 1 shows the demographic information for the two experimental and control groups. There was no significant statistical difference in terms of demographic information between the experimental and control groups. Before the intervention, the mean $\pm S D$ values of $\mathrm{HbA}_{1 \mathrm{c}}$ among the participants of the experimental and control groups were $7.80 \pm 1.09$ and $7.90 \pm 1.14$, respectively, and the difference between them was not statistically significant $(p>0.05)$. After the intervention, the mean $\pm \mathrm{SD}$ values of $\mathrm{HbA}_{1 \mathrm{c}}$ among the experimental and control groups became $7.03 \pm 0.88$ and $7.94 \pm 1.12$, respectively, with a significant statistical difference $(p<0.001)$. The results indicate an improvement in the amount of $\mathrm{HbA}_{1 c}(p<0.001)$ in the experimental group. However, it did not change significantly in patients in the control group.

Before the intervention, the mean \pm SD values of depression in the experimental and control groups were $18.58 \pm 9.59$ and $18.00 \pm 8.86$, respectively, and the difference between them was not statistically significant $(p>0.05)$. After the intervention, the mean $\pm S D$ values of depression among the participants in the experimental and control groups became $4.73 \pm 1.97$ and $18.34 \pm 8.88$, respectively, with a significant statistical difference $(p<0.001)$. The results indicated a significant reduction in the level of depression $(p<0.001)$ after the educational intervention in the experimental group. However, the rate of depression in patients in the control group did not change significantly.

Table 2 displays the level of overt and covert anxiety in the experimental and control groups separately. As a result, the level of overt and covert anxiety in the experimental group $(p<0.001)$ was significantly reduced. Table 3 shows the frequency of distribution of overt anxiety, and Table 4 represents the frequency of distribution of covert anxiety. Table 5 also shows the frequency of distribution of depression in patients before and after intervention, and the rate of overt and covert anxiety in most patients before the intervention was "moderate to high". The levels of depression in most patients were "relatively depressed", "need counseling" and "low depressed" before the intervention, but after the intervention, the prevalence of overt and covert anxiety was "moderate to low" and "mild"; the rate of depression also changed to "natural".

\begin{tabular}{|c|c|c|c|c|c|}
\hline \multirow[t]{2}{*}{ Subtype } & \multirow[t]{2}{*}{ Demographic variables } & \multicolumn{3}{|l|}{ Frequency (\%) } & \multirow[t]{2}{*}{$p$} \\
\hline & & $\begin{array}{l}\text { Interventional } \\
\text { group }\end{array}$ & Control group & Total & \\
\hline \multirow[t]{2}{*}{ Gender } & male & 29 (39.7) & $27(37.0)$ & $56(38.4)$ & \multirow[t]{2}{*}{0.734} \\
\hline & female & $44(60.3)$ & $73(63.0)$ & $90(61.6)$ & \\
\hline \multirow[t]{3}{*}{ Level of education } & $<$ diploma & $40(54.8)$ & $31(42.5)$ & $71(48.6)$ & \multirow[t]{3}{*}{0.192} \\
\hline & diploma & $27(37.0)$ & $30(41.1)$ & $57(39.0)$ & \\
\hline & > diploma & $6(8.2)$ & $12(16.4)$ & $18(12.3)$ & \\
\hline \multirow[t]{5}{*}{ Occupational status } & housewife & $37(51.4)$ & $31(42.5)$ & $68(46.9)$ & \multirow[t]{5}{*}{0.568} \\
\hline & unemployed & $7(9.7)$ & $8(11.0)$ & $15(10.3)$ & \\
\hline & manual worker & $13(18.1)$ & $14(19.2)$ & $27(18.6)$ & \\
\hline & employee & $10(13.9)$ & $17(23.3)$ & $27(18.6)$ & \\
\hline & retired & $5(6.9)$ & $3(4.1)$ & $8(5.5)$ & \\
\hline \multirow[t]{3}{*}{ Income } & one million & $36(49.3)$ & $31(42.5)$ & $67(45.9)$ & \multirow[t]{3}{*}{0.476} \\
\hline & between one and three million & $31(42.5)$ & $38(52.1)$ & $69(47.3)$ & \\
\hline & more than three million & $6(8.2)$ & $4(5.5)$ & $10(6.8)$ & \\
\hline \multirow[t]{5}{*}{ Admissions } & once & $17(23.3)$ & $22(30.1)$ & $39(26.7)$ & \multirow[t]{5}{*}{0.667} \\
\hline & twice & $20(27.4)$ & $22(30.1)$ & $42(28.8)$ & \\
\hline & three times & $12(16.4)$ & $11(15.1)$ & $23(15.8)$ & \\
\hline & more than three times & $20(27.4)$ & $13(17.8)$ & $33(22.6)$ & \\
\hline & never & $4(5.5)$ & $5(6.8)$ & $9(6.2)$ & \\
\hline \multirow[t]{4}{*}{ Anxiety history } & divorce & $9(12.3)$ & $6(8.2)$ & $15(10.3)$ & \multirow[t]{4}{*}{0.396} \\
\hline & family members with disease & $9(12.3)$ & $5(6.8)$ & $14(9.6)$ & \\
\hline & death of family members & $5(6.8)$ & $9(12.3)$ & $14(9.6)$ & \\
\hline & none & $50(68.5)$ & $53(72.6)$ & $103(70.5)$ & \\
\hline \multirow[t]{2}{*}{ Age } & \multirow[t]{2}{*}{$M \pm S D$} & \begin{tabular}{|l|} 
experimental \\
group
\end{tabular} & $3.67 \pm 0.7$ & & \multirow[t]{2}{*}{0.386} \\
\hline & & control group & $3.57 \pm 0.62$ & & \\
\hline
\end{tabular}

\begin{tabular}{|c|c|c|c|c|c|c|}
\hline \multicolumn{2}{|c|}{ Group Time } & Before & After & $M \pm$ Difference & $t$ & $p$ \\
\hline \multirow[t]{3}{*}{ State (apparent) anxiety } & case & $48.94 \pm 8.92$ & $30.30 \pm 2.24$ & $-18.64 \pm 8.34$ & 19.07 & $<0.001$ \\
\hline & control & $48.61 \pm 8.53$ & $48.79 \pm 8.31$ & $0.17 \pm 0.78$ & 1.93 & 0.057 \\
\hline & $p$ & 0.820 & $<0.001$ & $<0.001$ & & \\
\hline \multirow[t]{3}{*}{ Trait (hidden) anxiety } & case & $44.37 \pm 7.24$ & $25.97 \pm 2.71$ & $-18.40 \pm 6.36$ & 24.54 & $<0.001$ \\
\hline & control & $43.57 \pm 8.84$ & $44.17 \pm 8.47$ & $0.60 \pm 1.35$ & 3.81 & $<0.001$ \\
\hline & $p$ & 0.663 & $<0.001$ & $<0.001$ & & \\
\hline
\end{tabular}




\begin{tabular}{|l|l|l|l|}
\hline \multicolumn{3}{|l|}{ Table 3. Prevalence of State (apparent) anxiety } \\
\hline \multirow{4}{*}{ Group } & Categorization & \multicolumn{2}{l|}{ Frequency (\%) } \\
\cline { 3 - 4 } & & Before & After \\
\hline Experimental group & mild & $2(7.2)$ & $48(8.65)$ \\
\cline { 2 - 4 } & medium to low & $13(8.17)$ & $25(2.34)$ \\
\cline { 2 - 4 } & medium to high & $38(1.2)$ & \\
\cline { 2 - 4 } & fairly intense & $18(7.24)$ & \\
\cline { 2 - 4 } & intense & $2(7.2)$ & $4(5.5)$ \\
\hline Control group & mild & $6(2.8)$ & $6(2.8)$ \\
\hline & medium to low & $5(8.6)$ & $46(63)$ \\
\cline { 2 - 4 } & medium to high & $45(6.61)$ & $15(5.20)$ \\
\cline { 2 - 4 } & fairly intense & $15(5.20)$ & $2(7.2)$ \\
\cline { 2 - 4 } & intense & $2(7.2)$ & \\
\hline
\end{tabular}

\begin{tabular}{|l|l|l|l|}
\hline \multicolumn{4}{|l|}{ Table 4. Prevalence of Trait (hidden) anxiety } \\
\hline \multirow{4}{*}{ Group } & Time & Categorization & Frequency (\%) \\
\cline { 3 - 4 } & & Before & After \\
\hline \multirow{5}{*}{ Experimental group } & mild & $3(1.4)$ & $71(6.98)$ \\
\cline { 2 - 4 } & medium to low & $28(8.34)$ & $1(4.1)$ \\
\cline { 2 - 4 } & medium to high & $32(8.43)$ & \\
\cline { 2 - 4 } & fairly intense & $10(7.13)$ & \\
\hline Control group & mild & $8(11)$ & $5(8.6)$ \\
\cline { 2 - 4 } & medium to low & $26(6.35)$ & $28(4.38)$ \\
\cline { 2 - 4 } & medium to high & $31(5.42)$ & $31(5.42)$ \\
\cline { 2 - 4 } & fairly intense & $7(6.9)$ & $8(11)$ \\
\cline { 2 - 4 } & intense & $1(4.1)$ & $1(4.1)$ \\
\hline
\end{tabular}

\begin{tabular}{|c|c|c|c|}
\hline \multirow[b]{2}{*}{ Group } & \multirow[t]{2}{*}{ Categorization } & \multicolumn{2}{|c|}{ Frequency (\%) } \\
\hline & & Before & After \\
\hline \multirow[t]{6}{*}{ Experimental group } & normal & $11(1.15)$ & $71(100)$ \\
\hline & mild depressed & $25(2.34)$ & \\
\hline & need counseling & $10(7.3)$ & \\
\hline & relatively depressed & $19(26)$ & \\
\hline & major depressed & $5(8.6)$ & \\
\hline & excessive depressed & $3(1.4)$ & \\
\hline \multirow[t]{6}{*}{ Control group } & normal & $14(2.19)$ & $13(8.17)$ \\
\hline & mild depressed & $23(5.31)$ & $24(9.32)$ \\
\hline & need counseling & $16(9.21)$ & $15(5.20)$ \\
\hline & relatively depressed & $14(2.19)$ & $14(2.19)$ \\
\hline & major depressed & $3(1.4)$ & $4(5.5)$ \\
\hline & excessive depressed & $3(1.4)$ & $3(0.4)$ \\
\hline
\end{tabular}

\section{Discussion}

This study aimed to investigate the effect of spiritual education on anxiety and depression and better control of blood sugar in patients with type 2 diabetes mellitus. The results of the study showed a significant decrease in the level of depression and overt and covert anxiety in the experimental group compared to the control group after four sessions of spiritual training. The level of overt and covert anxiety was moderate to high before the intervention, but anxiety in the experimental group was mild and moderate to low after the intervention. The level of depression was also mild to relatively depressed before the intervention, but after the intervention, the level of depression changed to normal. The rate of blood sugar control, which was checked with the $\mathrm{HbA}_{1 \mathrm{c}}$ index, improved to the desired level after the intervention. Therefore, it can be concluded that spiri- tual training is a good way to reduce anxiety and depression. It can also help one to have better control over blood sugar levels. The results of many studies are consistent with the present study and have shown the positive effect of spiritual interventions. A study in 2019 revealed that spiritual care based on the "Ghalbe Salim" model could reduce the state and trait anxiety of mothers having premature neonates [24]. The results of studies by Kandasamy et al. (2011) and Amiri et al. (2018) showed that spiritual education in cancer patients can reduce stress, anxiety and depression $[25,26]$. Some studies have also revealed that the treatment of depression and anxiety in patients with type 2 diabetes mellitus was facilitated by combining spiritual values and patients' beliefs [27, 28]. The study by Newlin et al. in 2008 revealed that spirituality and religiosity played a significant role in controlling the patients' blood sugar; this is in line with the results of the present study [29]. Therefore, according to these results, it can be concluded that spiritual training can be used in 
improving life, especially in terms of stress - including depression and anxiety - and in controlling blood sugar levels. On the other hand, in the study of Zareipour et al., which was carried out in 2016, there was no relationship between mental health and blood sugar control [30], and in the study by Long et al. in 2018, there was no significant relationship between spirituality and control of body mass index and increased physical activity [31]. Different samples and cultural differences may explain the discrepancies. However, as mentioned, spiritual training can improve mental health and the control of blood sugar levels. In the present study, the patients were also followed for four months, and the difference in the results of this study can be attributed to the difference in interventions. Nurses and other health care providers must consider the spiritual dimension of health for empowering individuals and communities, especially patients suffering from chronic diseases such as diabetes, to achieve health and comprehensive well-being [32].

\section{Limitations of the study}

The limitations of this study are the small number of people studied and the lack of access to people of different religions. Therefore, it is recommended that this research be carried out with a larger number of individuals and include other religions and denominations, even if other smaller studies have been carried out, as well as evaluating the long-term results.

\section{Conclusions}

As the results of this study showed, spiritual education is an effective way to help patients with type 2 diabetes mellitus, especially when it comes to depression, anxiety and blood sugar control. These findings are important in reducing psychological problems and improving disease management in individual with diabetes. Therefore, spiritual education as part of diabetes care programs should be considered by health care providers. It is recommended that the necessary steps should be taken to teach spiritual concepts to patients with diabetes.

Acknowledgements. This article is the result of a research project approved by the Shiraz University of Medical Sciences, number: 96-08-86-16361 and ethical code: IR.SUMS.REC.1397.214 and IRCT20090908002432N7. We would hereby like to thank the Shiraz University of Medical Sciences for financial support, the personnel of the Emam Reza Clinic, affiliated with the Shiraz University of Medical Sciences, and all the patients who participated and cooperated in the study at the Emam Reza Clinic. We would also like to thank Dr. Nasrin Shokrpour at the Clinical Research Development Center of Nemazee Hospital, Shiraz University of Medical Sciences, and Rozhan Ghobadimoghaddam at the Middle East Technical University, Ankara, Turkey, for editorial assistance.

Source of funding: This work was supported in part by the Research Deputy of the Shiraz University of Medical Sciences, Shiraz, Iran. Conflicts of interest: The authors declare no conflicts of interest.

\section{References}

1. Martino G, Caputo A, Bellone F, et al. Going beyond the visible in type 2 diabetes mellitus: defense mechanisms and their associations with depression and health-related quality of life. Front Psychol 2020; 11: 267, doi: 10.3389/fpsyg.2020.00267.

2. Cho N, Shaw J, Karuranga S, et al. IDF Diabetes Atlas: Global estimates of diabetes prevalence for 2017 and projections for 2045. Diabetes Res Clin Pract 2018; 138: 271-281, doi: 10.1016/j.diabres.2018.02.023.

3. Esteghamati A, Etemad K, Koohpayehzadeh J, et al. Trends in the prevalence of diabetes and impaired fasting glucose in association with obesity in Iran: 2005-2011. Diabetes Res Clin Pract 2014; 103(2): 319-327, doi 10.1016/j.diabres.2013.12.034.

4. Sadock BJ, Sadock VA, Kaplan HI. Kaplan and Sadock's concise textbook of child and adolescent psychiatry. Philadelphia: Lippincott Williams \& Wilkins; 2009.

5. Semenkovich K, Brown ME, Svrakic DM, et al. Depression in type 2 diabetes mellitus: prevalence, impact, and treatment. Drugs 2015; 75(6): 577-587, doi: 10.1007/s40265-015-0347-4.

6. Lustman PJ, Clouse RE. Depression in diabetic patients: the relationship between mood and glycemic control. J Diabetes Complications 2005; 19(2): 113-122, doi: 10.1016/j.jdiacomp.2004.01.002.

7. Sun N, Lou P, Shang Y, et al. Prevalence and determinants of depressive and anxiety symptoms in adults with type 2 diabetes in China: a cross-sectional study. BMJ Open 2016; 6(8): e012540, doi: 10.1136/bmjopen-2016-012540.

8. Hofmeijer-Sevink MK, Batelaan NM, Megen HJ van, et al. Clinical relevance of comorbidity in anxiety disorders: a report from the Netherlands Study of Depression and Anxiety (NESDA). J Affect Disord 2012; 137(1-3): 106-112, doi: 10.1016/j.jad.2011.12.008.

9. Alizadeh Moghdam A, Safari AR, Feizi M. The relationship between anxiety, depression and stress with the severity of diabetes: the role of the mediator of quality of life. IJDLD 2019; 18(2): 80-89.

10. Koenig HG. Religion, spirituality, and medicine: research findings and implications for clinical practice. South Med J 2004; 97(12): 1194-1200.

11. Vizehfar F, Jaberi A. The relationship between religious beliefs and quality of life among patients with multiple sclerosis. J Relig Health 2017; 56(5): 1826-1836, doi: 10.1007/s10943-017-0411-3.

12. Parsian NDT. Spirituality and coping in young adults with diabetes: a cross-sectional study. Eur Diabet Nurs 2009; 6(3): 100-104, doi: 10.1002/edn.144.

13. Braam AW, Schrier AC, Tuinebreijer WC, et al. Religious coping and depression in multicultural Amsterdam: a comparison between native Dutch citizens and Turkish, Moroccan and Surinamese/Antillean migrants. J Affect Ddisord 2010; 125(1-3): 269-278, doi: 10.1016/j.jad.2010.02.116.

14. Starczewska M, Kujawska R, Stanisławska $M$, et al. The analysis of health behaviors and illness acceptance in patients with diabetes. Fam Med Prim Care Rev 2018; 20(4): 352-355, doi: 10.5114/fmpcr.2018.79347.

15. Fitchett G, Murphy PE, Kim J, et al. Religious struggle: prevalence, correlates and mental health risks in diabetic, congestive heart failure, and oncology patients. Int J Psychiatry Med 2004; 34(2): 179-196, doi: 10.2190/UCJ9-DP4M-9C0X-835M.

16. Lundberg PC, Thrakul S. Religion and self-management of Thai Buddhist and Muslim women with type 2 diabetes. J Clin Nurs 2013; 22(13-14): 1907-1916, doi: 10.1111/jocn.12130.

17. Kharatzadeh $\mathrm{H}$, Davazdahemami $\mathrm{M}$, Bakh $\mathrm{M}$, et al. Effectiveness of mindfulness based stress reduction on glycemic control, stress, anxiety and depression on patients with type 2 diabetes mellitus. Urmia Med J 2017; 28(3): 207-214.

18. Zsido AN, Teleki SA, Csokasi K, et al. Development of the short version of the Spielberger State-Trait Anxiety Inventory. Psychiatry Res 2020: 113223, doi: 10.1016/j.psychres.2020.113223.

19. Panahi Shahri M. Preliminary evaluation of validity \& reliability of Spielberger state-trait anxiety inventory [MA thesis]. Tehran: Tarbiat Modarres University; 1993. 
20. Mahram B. Standardization of Spielberger inventory in Mashhad [MA thesis]. Tehran: Allameh Tabatabaee University; 1994.

21. Beck AT, Steer RA, Carbin MG. Psychometric properties of the Beck Depression Inventory: twenty-five years of evaluation. Clin Psychol Rev 1988; 8(1): 77-100, doi: 10.1016/0272-7358(88)90050-5.

22. Azkhosh M. Application of psychological tests and clinical diagnosis. Tehran: Ravan Publication; 2008.

23. Bukhsh A, Khan TM, Nawaz MS, et al. Association of diabetes knowledge with glycemic control and self-care practices among Pakistani people with type 2 diabetes mellitus. Diabetes Metab Syndr Obes Targets Ther 2019; 12: 1409, doi: 10.2147/DMSO.S209711.

24. Edraki M, Noeezad Z, Bahrami R, et al. Effect of spiritual care based on "Ghalbe Salim" model on anxiety among mothers with premature newborns admitted to neonatal intensive care units. IJN 2019; 10(1): 50-57, doi: 10.22038/IJN.2018.31210.1428.

25. Kandasamy A, Chaturvedi S, Desai G. Spirituality, distress, depression, anxiety, and quality of life in patients with advanced cancer. Indian J Cancer 2011; 48(1): 55-59.

26. Amiri A, Mostafapour Z, Nassabeh F, et al. The effect of spiritual education on stress, anxiety and depression in patients with Breast Cancer Referred to Shahid Motahari Clinic in Shiraz in 2018. Journal of Research on the Lepidoptera 2020; 51(1): 60-73.

27. Lynch CP, Hernandez-Tejada MA, Strom JL, et al. Association between spirituality and depression in adults with type 2 diabetes. Diabetes Educ 2012; 38(3): 427-435.

28. Niaz Azari M, Abdollahi M, Zabihi Hesari NK, et al. Effect of spiritual group therapy on anxiety and quality of life among gestational diabetic females. JRH 2017; 5(1): 11-20.

29. Newlin K, Melkus GD, Tappen R, et al. Relationships of religion and spirituality to glycemic control in Black women with type 2 diabetes. Nurs Res 2008; 57(5): 331-339, doi: 10.1097/01.NNR.0000313497.10154.66

30. Zareipour M, Mahmoodi H, Valizadeh R, et al. The association between spiritual health and blood sugar control in elderly patients with type 2 diabetes. Elderly Health J 2016; 2(2): 67-72.

31. Long SEP. Diabetes Prevention Program: exploring spirituality and spiritual interventions on outcomes. Kalamazoo: Western Michigan University, An Arbor: ProQuest Dissertations Publishing; 2018.

32. Jaberi A, Momennasab M, Cheraghi M, et al. Spiritual health as experienced by muslim adults in Iran: a qualitative content analysis. Shiraz E-Medical Journal 2019; 20(12), doi: 10.5812/semj.88715.

Tables: 5

Figures: 0

References: 32

Received: 10.07.2020

Reviewed: 31.10 .2020

Accepted: 04.11 .2020

Address for correspondence:

Azadeh Amiri, MSc

Fatemeh (PUBH) Nursing Midwifery School

Namazee Square

Postal code: 71936-13119 Shiraz

Iran

Tel.: +98 7136474254

E-mail: amiriea@sums.ac.ir 